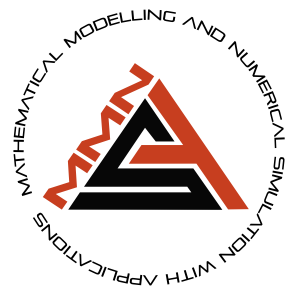

\title{
Flip and generalized flip bifurcations of a two-dimensional discrete-time chemical model
}

\author{
Parvaiz Ahmad Naik@1,*,‡ , Zohreh Eskandari $2,{ }^{\ddagger}$ and Hossein Eskandari \\ Shahraki@3, ${ }^{\ddagger}$
}
${ }^{1}$ School of Mathematics and Statistics, Xi'an Jiaotong University, Xi'an, Shaanxi 710049, P. R. China, ${ }^{2}$ Department of Mathematical Sciences, Shahrekord University, P.O. Box 115, Shahrekord, Iran, ${ }^{3}$ Amirkabir University of Technology, Faculty of Physics and Energy Engineering, No. 350, Hafez Ave, Valiasr Square, Tehran, Iran
*Corresponding Author
†naik.parvaiz@xjtu.edu.cn (Parvaiz Ahmad Naik); z.eskandari@sku.ac.ir (Zohreh Eskandari); hes30377@aut.ac.ir (Hossein Eskandari Shahraki)

\begin{abstract}
This paper focuses on introducing a two-dimensional discrete-time chemical model and the existence of its fixed points. Also, the one and two-parameter bifurcations of the model are investigated. Bifurcation analysis is based on numerical normal forms. The flip (period-doubling) and generalized flip bifurcations are detected for this model. The critical coefficients identify the scenario associated with each bifurcation. To confirm the analytical results, we use the MATLAB package MatContM, which performs based on the numerical continuation method. Finally, bifurcation diagrams are presented to confirm the existence of flip (period-doubling) and generalized flip bifurcations for the glycolytic oscillator model that gives a better representation of the study.
\end{abstract}

Key words: Bifurcation; normal form; numerical continuation method; one-parameter bifurcation; two-parameter bifurcation AMS 2020 Classification: 00A71; 80A30; $92 \mathrm{C} 45$

\section{Introduction}

Mathematical modelling is a powerful tool for understanding, designing, and predicting processes and process equipment in the chemical industry, including the conservation of momentum, energy, and material. Detailed modelling of complex reaction systems is becoming increasingly important in the development, analysis, design, and control of chemical reaction processes [1]. Numerical computer modelling can describe chemical reactions, composition, fluid flow, and temperature distribution in three dimensions and time. Chemical engineers frequently used fundamental dynamic models to develop new chemical processes and to predict the behavior of existing industrial processes accurately. For example, models can be used to simulate how a process will behave under new operating conditions, to indicate how new products can be made using an existing plant, to investigate product quality enhancements, and to achieve production rate improvements [2]. The systems of ordinary differential equations (ODEs) are involved in modelling the dynamics of reaction networks to track the time evolution of chemical concentrations for the species in the network. Through the differential equations using the theoretical results of dynamical systems, or numerical simulations, the study of the properties of the dynamics (e.g. stability of steady states, the existence of multiple steady states, etc.) are obtained [3].

Carden et al. [3] introduced a set of mathematical techniques for describing and characterizing a series of chemical processes (enzymesubstrate, protein-protein, etc.) that cells used to sense and respond to diverse stimuli during the progression and cellular behavior of cancers. Mahdy et al. [4], in their paper, combined the Sumudu decomposition method and the Hermite collocation method for the solutions

> Received: 06.11.2021 > Revised: 16.12.2021 > Accepted: 17.12.2021 > Published: 18.12.2021 
of a nonlinear biochemical reaction model. They have represented the signal flow graph and Simulink of MATLAB of the model in the paper. Besides, the stability of the equilibrium point was also studied. They show that the Sumudu decomposition method and Hermite collocation method are extremely symmetrical and similar. Apart from modelling chemical processes, mathematical modelling is not limited to any particular field but finds applications in different fields $[5,6,7,8,9,10,11,12,13,14,15,16,17,18]$. Alidousti et al. [19] studied the dynamic behaviors of the discrete Bonhoeffer-van der Pol (BVP) model. Through their results, It was shown that the BVP model undergoes codimension one (codim-1) bifurcations such as pitchfork, fold, flip (period-doubling), and Neimark-Sacker. Instead of codimension one (codim-1) bifurcations, the codimension two (codim-2) bifurcations including resonance 1:2, 1:3, 1:4, and Chenciner were also achieved.

Bifurcation analysis can divide the parameter space into more regions and depict more complex dynamic behaviors. Bifurcation analysis traces time-varying change(s) at a particular state of the system in a multidimensional space where each dimension represents a particular concentration of the biochemical factor involved. It can happen that a slight variation in a parameter can have a significant impact on the solution. Bifurcation analysis finds application in different fields. Ghori et al. [20], in their paper, studied the global dynamics and bifurcation analysis of a fractional-order SEIR epidemic model with a saturation incidence rate. The outcome of their study reveals that the model undergoes a transcritical bifurcation and a Hopf bifurcation at the equilibrium points under certain conditions for all model parameters at fractional-order $\alpha=1$. Wang and Jia [21] studied the stability and bifurcation analysis of a generalized Gray-Scott chemical reaction model. The results of their study show that the system exhibits abundant dynamical behaviors and the chemical reaction in the reactor will be in balance in the end under certain conditions. Khan [22], in his paper, studied the local dynamics and Neimark-Sacker bifurcation of a two-dimensional glycolytic oscillator model. It was found that the model has a unique equilibrium point for all $\alpha$ and $\beta$. Also, some bifurcation diagrams and the corresponding maximum Lyapunov exponent were presented for the model to verify the obtained results. Recently, Naik et al. [23] investigated the multiple bifurcations of a discrete-time prey-predator model with a mixed functional response. They detected the flip, Neimark-Sacker and strong resonance bifurcations of the model. The complex dynamical behavior of the model up to the $16^{\text {th }}$ iteration was investigated.

Although researchers tried to obtain the bifurcation results of the chemical model, none in the literature obtained the flip bifurcations of the model that motivates the authors to carry out this work and becomes the novelty of the present study. In this paper, we provide the dynamics of the glycolytic oscillator chemical model through the flip and generalized flip bifurcations analytically as well as numerically. Further, we calculate the critical coefficients of each bifurcation. The two-dimensional discrete-time chemical model under consideration is given as follows [22]

$$
\left\{\begin{array}{l}
x^{C h} \mapsto-x^{C h}\left(y^{C h}\right)^{2}-\beta x^{C h}+\alpha+x^{C h}, \\
y^{C h} \mapsto x^{C h}\left(y^{C h}\right)^{2}+\beta x^{C h}
\end{array}\right.
$$

where $x^{C h}$ and $y^{C h}$ are the substrate concentrations at time $t$ and $\alpha$ and $\beta$ are the dimensionless parameters.

The structure of the current study can be presented as follows. In Section 1, the introduction of the study is given. In Section 2, the analytical bifurcation results of the model are carried out in the form of theorems and proofs. Section 3 contains the numerical bifurcation analysis of the model to verify the analytical results. Finally, the concluding remarks about the proposed work are given in Section 4 . In the following section, we provide the existence of different types of bifurcations of the system (1).

\section{Bifurcation analysis}

Our first step is to obtain the fixed points of model (1) in order to investigate its bifurcations. Solving equations

$$
\begin{cases}-x^{C h}\left(y^{C h}\right)^{2}-\beta x^{C h}+\alpha+x^{C h} & =x^{C h} \\ x^{C h}\left(y^{C h}\right)^{2}+\beta x^{C h} & =y^{C h}\end{cases}
$$

yields that model (1) has a unique fixed point

$$
x_{*}^{C h}=\frac{\alpha}{\alpha^{2}+\beta}, \quad y_{*}^{C h}=\alpha .
$$

Analyzing the bifurcations of map

$$
\left(\begin{array}{l}
x^{C h} \\
y^{C h}
\end{array}\right) \mapsto M^{C h}(\mathcal{X}, \Lambda)=\left(\begin{array}{c}
-x^{C h}\left(y^{C h}\right)^{2}-\beta x^{C h}+\alpha+x^{C h} \\
x^{C h}\left(y^{C h}\right)^{2}+\beta x^{C h}
\end{array}\right)
$$

is discussed in this section, where $\mathcal{X}=\left(x^{C h}, y^{C h}\right)^{T}$ and $\Lambda=(\alpha, \beta)^{T}$.

Jacobian matrix, second-order multi-linear form, and third-order multi-linear form of (2) are as follows:

$$
\begin{aligned}
A^{C h}(\mathcal{X}, \Lambda) & =\left(\begin{array}{cc}
-\left(y^{C h}\right)^{2}-\beta+1 & -2 x^{C h} y^{C h} \\
\left(y^{C h}\right)^{2}+\beta & 2 x^{C h} y^{C h}
\end{array}\right), \\
B^{C h}\left(\widehat{X^{C h}}, \widehat{Y^{C h}}\right) & =\left(\begin{array}{c}
-2 y^{C h}\left(x_{1} y_{2}+x_{2} y_{1}\right)-2 x^{C h} x_{2} y_{2} \\
2 y^{C h}\left(x_{1} y_{2}+x_{2} y_{1}\right)+2 x^{C h} x_{2} y_{2}
\end{array}\right),
\end{aligned}
$$




$$
C^{C h}\left(\widehat{X^{C h}}, \widehat{Y^{C h}}, \widehat{Z^{C h}}\right)=\left(\begin{array}{c}
-2 x_{1} y_{2} z_{2}-2 x_{2} y_{1} z_{2}-2 x_{2} y_{2} z_{1} \\
2 x_{1} y_{2} z_{2}+2 x_{2} y_{1} z_{2}+2 x_{2} y_{2} z_{1}
\end{array}\right)
$$

where $\widehat{X^{C h}}=\left(x_{1}, x_{2}\right)^{T}, \widehat{Y^{C h}}=\left(y_{1}, y_{2}\right)^{T}$ and $\widehat{Z^{C h}}=\left(z_{1}, z_{2}\right)^{T}$.

Flip bifurcation of $\mathcal{X}_{*}^{\text {Ch }}$

The bifurcation parameter $\beta$ and the fixed parameter $\alpha$ are considered here.

Theorem 1 At $\alpha=\alpha_{P D}=-\alpha^{2}+1+\sqrt{4 \alpha^{2}+1}$, a flip bifurcation occurs for $\mathcal{X}_{*}^{C h}$.

Proof As we can see, the multipliers of

$$
A^{C h}\left(\mathcal{X}_{*}^{C h}, \wedge_{P D}\right)=\left(\begin{array}{cc}
-\sqrt{4 \alpha^{2}+1} & -2 \frac{\alpha^{2}}{1+\sqrt{4 \alpha^{2}+1}} \\
1+\sqrt{4 \alpha^{2}+1} & 2 \frac{\alpha^{2}}{1+\sqrt{4 \alpha^{2}+1}}
\end{array}\right), \quad \wedge_{P D}=\left(\alpha, \beta_{P D}\right)^{T},
$$

where $\wedge_{P D}=\left(\alpha_{P D}, \beta\right)^{T}$, are the following

$$
\lambda_{1}^{P D}=-1, \quad \lambda_{2}^{P D}=-1 / 2 \sqrt{4 \alpha^{2}+1}+1 / 2 .
$$

If $\lambda_{2}^{P D} \neq \pm 1, A^{C h}\left(\mathcal{X}_{*}^{C h}, \wedge_{P D}\right)$ has a simple multiplier -1 on the unit circle. So $M^{C h}\left(\mathcal{X}_{*}^{C h}, \wedge_{P D}\right)$ is possible to write as

$$
\eta \mapsto-\eta+\widehat{b_{P D}} \eta^{3}+\mathcal{O}\left(\eta^{4}\right),
$$

where

$$
\left.\widehat{b_{P D}}=\frac{1}{6}\left\langle w_{P D}, C^{C h}\left(v_{P D}, v_{P D}, v_{P D}\right)+3 B^{C h}\left(v_{P D},\left(I_{2}-A^{C h}\left(\mathcal{X}_{*}^{C h}, \wedge_{P D}\right)\right)\right)^{-1} B^{C h}\left(v_{P D}, v_{P D}\right)\right)\right\rangle,
$$

and

$$
A^{C h}\left(\mathcal{X}_{*}^{C h}, \wedge_{P D}\right) v_{P D}=-v_{P D}, \quad\left(A^{C h}\left(\mathcal{X}_{*}^{C h}, \wedge_{P D}\right)\right)^{T} w_{P D}=-w_{P D}, \quad\left\langle w_{P D}, v_{P D}\right\rangle=1 .
$$

As a result

$$
v_{P D}=\left(\begin{array}{c}
-1 / 2 \\
1
\end{array}\right), \quad w_{P D}=\left(\begin{array}{c}
2 \frac{1+\sqrt{4 \alpha^{2}+1}}{\sqrt{4 \alpha^{2}+1}-3} \\
2 \frac{\sqrt{4 \alpha^{2}+1}-1}{\sqrt{4 \alpha^{2}+1}-3}
\end{array}\right)
$$

and

$$
\widehat{b_{P D}}=-4 \frac{2 \alpha^{2} \sqrt{4 \alpha^{2}+1}-2 \alpha^{2}-\sqrt{4 \alpha^{2}+1}-1}{\left(1+\sqrt{4 \alpha^{2}+1}\right)^{2}\left(\sqrt{4 \alpha^{2}+1}-3\right)}
$$

$\widehat{b_{P D}} \neq 0$ yields a generic bifurcation. This bifurcation is supercritical (subcritical) if $\widehat{b_{P D}}>0\left(\widehat{b_{P D}}<0\right)$ and 2-period points bifurcated from $\mathcal{X}_{*}^{\text {Ch }}$ are stable (unstable). For more details see [24, 25, 26].

Generalized flip bifurcation of $\mathcal{X}_{*}^{\text {Ch }}$

The bifurcation parameters $\beta$ and $\alpha$ are considered here.

Theorem 2 At $\alpha=\alpha_{G P D}=1 / 2 \sqrt{2+2 \sqrt{2}}$ and $\beta=\beta_{G P D}=3 / 2+1 / 2 \sqrt{2}$, a generalized flip bifurcation occurs for $\mathcal{X}_{*}^{C h}$.

Proof Based on an assumption

$$
\alpha=\alpha_{G P D}=1 / 2 \sqrt{2+2 \sqrt{2}}, \quad \beta=\beta_{G P D}=3 / 2+1 / 2 \sqrt{2},
$$

the Jacobian matrix

$$
A^{C h}\left(\mathcal{X}_{*}^{C h}, \wedge_{G P D}\right)=\left(\begin{array}{cc}
-1-\sqrt{2} & -\frac{\sqrt{2}+1}{2+\sqrt{2}} \\
2+\sqrt{2} & \frac{\sqrt{2}+1}{2+\sqrt{2}}
\end{array}\right), \quad \wedge_{G P D}=\left(\alpha_{P D}, \beta_{P D}\right)^{T},
$$


has a simple critical multiplier $\lambda_{1}^{G P D}=1$, and no other multiplier is not on the unit circle and $\widehat{b_{P D}}=0$. So $M^{C h}\left(\mathcal{X}_{*}^{C h}, \wedge_{G P D}\right)$ is possible to write as

$$
\eta \mapsto-\eta+\widehat{c_{G P D}} \eta^{5}+\mathcal{O}\left(\eta^{6}\right)
$$

where

$$
\begin{aligned}
\widehat{c_{G P D}} & =\frac{1}{120}\left\langle w_{G P D}, 5 B^{C h}\left(v_{G P D}, h_{4}^{C h}\right)+10 C^{C h}\left(v_{G P D}, v_{G P D}, h_{3}^{C h}\right)+10 B^{C h}\left(h_{2}^{C h}, h_{3}^{C h}\right)\right. \\
& \left.+15 C^{C h}\left(v_{G P D}, h_{2}^{C h}, h_{2}^{C h}\right)\right\rangle, \\
h_{2}^{C h} & =\left(I_{2}-A^{C h}\right)^{-1} B^{C h}\left(v_{G P D}, v_{G P D}\right), \\
h_{3}^{C h} & =-\left(A^{C h}+I_{2}\right)^{I N V}\left(C^{C h}\left(v_{G P D}, v_{G P D}, v_{G P D}\right)+3 B^{C h}\left(v_{G P D}, h_{2}^{C h}\right)\right), \\
h_{4}^{C h} & =\left(I_{2}-A^{C h}\right)^{-1}\left(4 B^{C h}\left(v_{G P D}, h_{3}^{C h}\right)+3 B^{C h}\left(h_{2}^{C h}, h_{2}^{C h}\right)+6 C^{C h}\left(v_{G P D}, v_{G P D}, h_{2}\right)\right),
\end{aligned}
$$

and

$$
A^{C h}\left(\mathcal{X}_{*}^{C h}, \wedge_{G P D}\right) v_{P D}=-v_{G P D}, \quad\left(A^{C h}\left(\mathcal{X}_{*}^{C h}, \wedge_{G P D}\right)\right)^{T} w_{G P D}=-w_{G P D}, \quad\left\langle w_{G P D}, v_{G P D}\right\rangle=1 .
$$

As a result

$$
\begin{aligned}
& v_{\mathrm{GPD}}=\left(\begin{array}{c}
-1 / 2 \\
1
\end{array}\right), \quad w_{\mathrm{GPD}}=\left(\begin{array}{c}
-\frac{(2+\sqrt{2}) \sqrt{2}}{\sqrt{2}-1} \\
-2(\sqrt{2}-1)^{-1}
\end{array}\right), \\
& h_{2}^{C h}=\left(\begin{array}{c}
\frac{\sqrt{2+2 \sqrt{2}}(\sqrt{2}+1)}{(2+\sqrt{2})^{2}} \\
0
\end{array}\right), \quad h_{3}^{C h}=\left(\begin{array}{l}
0 \\
0
\end{array}\right), \quad h_{4}^{C h}=\left(\begin{array}{c}
-12 \frac{\sqrt{2+2 \sqrt{2}}(\sqrt{2}+1)}{(2+\sqrt{2})^{3}} \\
0
\end{array}\right) .
\end{aligned}
$$

So $\widehat{C_{G P D}}$ can be obtained as follows:

$$
\widehat{C_{G P D}}=-1 \text {. }
$$

Since $\widehat{c_{G P D}}$, the generalized flip is generic.

\section{Numerical continuation of $M^{C h}(\mathcal{X}, \wedge)$}

To confirm the analytical results, we use MATCONTM, a toolbox of MATLAB and works based on the numerical continuation method, for more details, see [27, 28]. Here $\alpha$ and $\beta$ are considered as a free parameter and a fixed parameter, respectively. Here we consider $\alpha=1$, by varying $\beta$ the flip bifurcations occurs at $\mathcal{X}_{*}^{C h}=(.309017,1.00000)$ for $\beta \beta_{P D}=2.23606$ where $\widehat{b_{P D}}=-3.05573 \times 10^{-1}$. According to the sign of $\widehat{b_{P D}}$ result in the flip bifurcation is sub-critical. Continuation of $\mathcal{X}_{*}^{C h}$ in $\left(x^{C h}, \beta\right)$-space is shown in Figure 1.

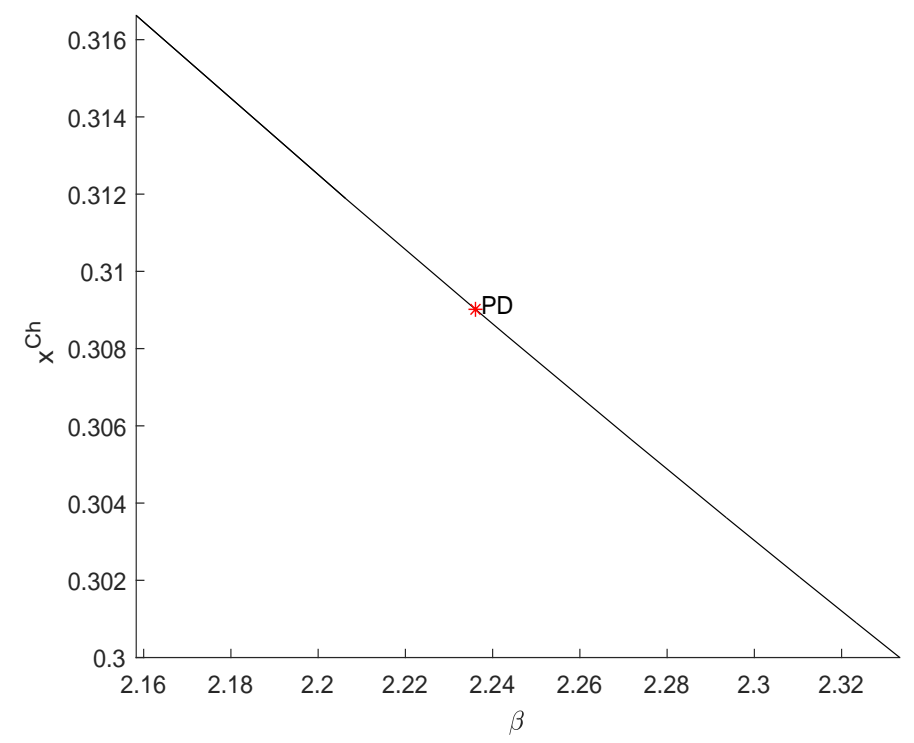

Figure 1. Continuation of $\mathcal{X}_{*}^{\text {Ch }}$ in $\left(\chi^{\text {Ch }}, \beta\right)$-space. 
Selecting this PD point and starting the continuation of a PD bifurcation curve in two control parameters $\alpha$ and $\beta$, yield a generalized flip bifurcation at $\mathcal{X}_{*}^{C h}=(0.321797,1.098684)$ for $\alpha=\alpha_{G P D}=1.098684$ and $\beta=\beta_{G P D}=2.207106$ with $\widehat{c_{G P D}}=-6.400000 \times 10^{-1}$, see Figure 2 .

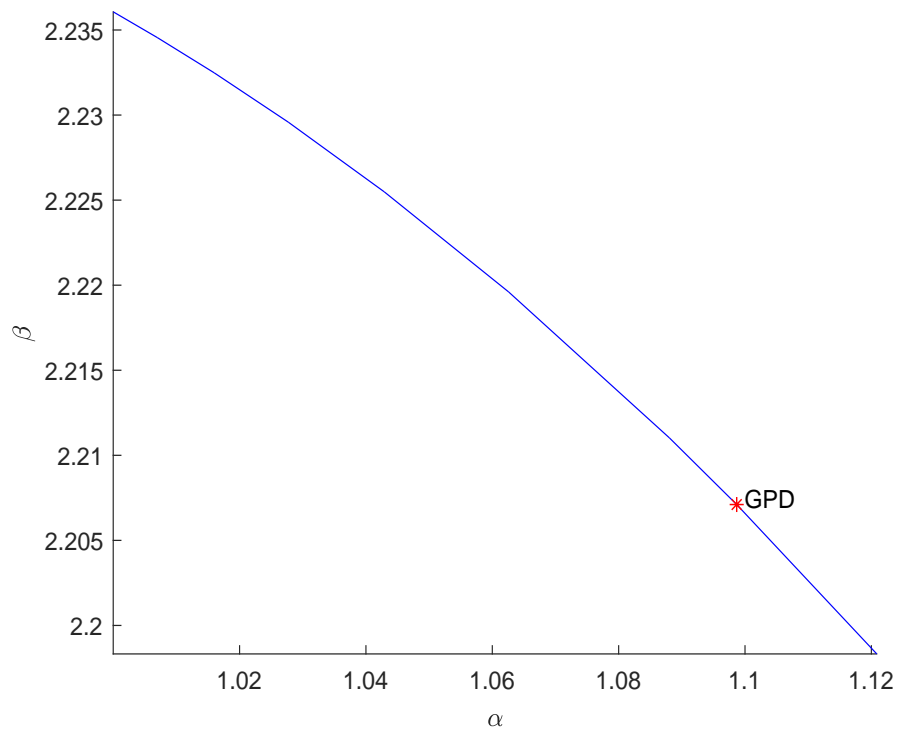

Figure 2. Flip bifurcation curve

We compute a branch of fold points of the second iterate by switching at the GPD point, see Figure 3.

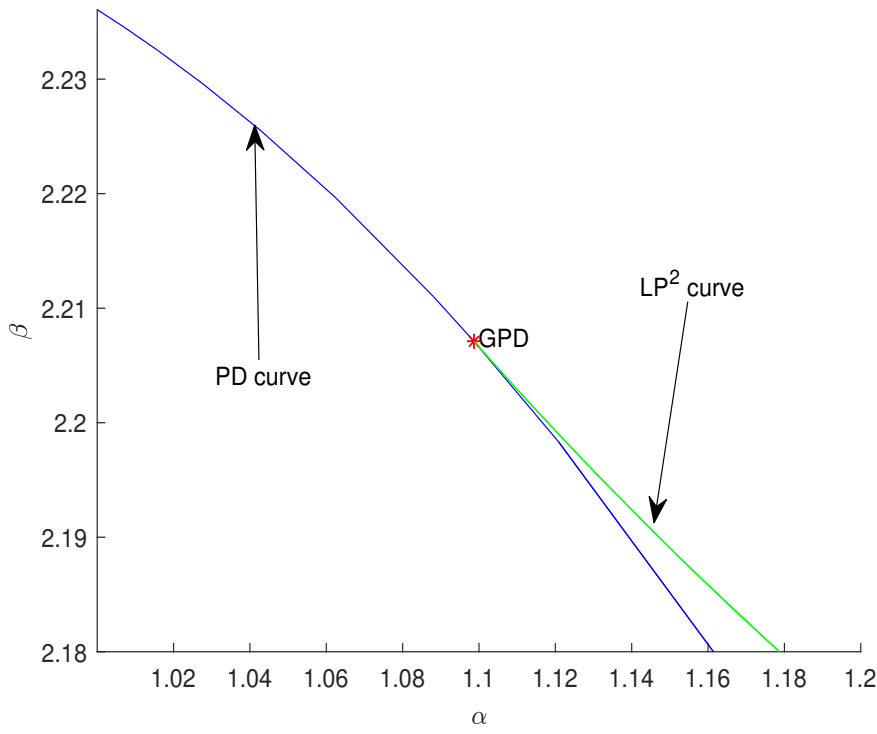

Figure 3. A curve of fold bifurcations of the second iterate, $L P^{2}$, which emanates tangentially at a GPD point on a flip curve.

\section{Conclusion}

In this paper, we provided the dynamics of the glycolytic oscillator chemical model through the flip and generalized flip bifurcations analytically as well as numerically. To investigate the bifurcations of this model, we calculated the critical coefficients of each bifurcation. These coefficients determined whether a bifurcation is non-degenerate and determined the scenario of each bifurcation. The results obtained in Sections 2 and 3 show excellent agreement between the analytical predictions and the numerical observations. From the obtained results, it is concluded that the model shows flip and generalized flip bifurcation indicating that the substrate concentrations vary from one period to another. Although the current paper studied a standard two-dimensional discrete-time chemical model but can be extended to fractional-order derivatives with the operators known as Caputo, Atangana-Gomez, Atangana-Baleanu, Caputo-Fabrizio, and others discover more causative factors that are not covered in this paper, such is left for future research direction. 


\section{Declarations}

\section{Consent for publication}

Not applicable.

\section{Conflicts of interest}

The authors declare that they have no conflict of interests.

\section{Funding}

Not applicable.

\section{Author's contributions}

P.A.N.: Conceptualization, Methodology, Supervision, Investigation, Writing-Original draft. Z.E.: Software, Methodology, Data Curation, Writing-Original draft. H.E.S.: Visualization, Investigation, Software, Writing-Reviewing and Editing. All authors discussed the results and contributed to the final manuscript.

\section{Acknowledgements}

Not applicable.

\section{References}

[1] Okino, M.S. \& Mavrovouniotis, M.L. Simplification of mathematical models of chemical reaction systems. Chemical Reviews, 98(2), 391-408, (1998).

[2] McLean, K.A.P. \& McAuley, K.B. Mathematical modelling of chemical processes-obtaining the best model predictions and parameter estimates using identifiability and estimability procedures. Canadian Journal of Chemical Engineering, 90(2), 351-366, (2012). [CrossRef]

[3] Carden, J., Pantea, C., Craciun, G., Machiraju, R. \& Mallick, P. Mathematical methods for modeling chemical reaction networks. bioRxiv, 070326, (2016). [CrossRef]

[4] Mahdy, A.M.S. \& Higazy, M. Numerical different methods for solving the nonlinear biochemical reaction model.International Journal of Applied and Computational Mathematics, 5(6), 1-17, (2019). [CrossRef]

[5] Yavuz, M. \& Sene, N. Fundamental calculus of the fractional derivative defined with Rabotnov exponential kernel and application to nonlinear dispersive wave model.Journal of Ocean Engineering and Science, 6(2), 196-205, (2021). [CrossRef]

[6] Naik, P.A., Yavuz, M., Qureshi, S., Zu, J. \& Townley, S. Modeling and analysis of COVID-19 epidemics with treatment in fractional derivatives using real data from Pakistan. The European Physical Journal Plus, 135(10), 1-42, (2020). [CrossRef]

[7] Dasbasi, B. Stability analysis of an incommensurate fractional-order SIR model. Mathematical Modelling and Numerical Simulation with Applications (MMNSA), 1(1), 44-55, (2021). [CrossRef]

[8] Naik, P.A., Owolabi, K.M., Zu, J. \& Naik, M.U.D. Modeling the transmission dynamics of COVID-19 pandemic in Caputo type fractional derivative.Journal of Multiscale Modelling, 12(3), 2150006-107, (2021). [CrossRef]

[9] Hammouch, Z., Yavuz, M. \& Özdemir, N. Numerical solutions and synchronization of a variable-order fractional chaotic system. Mathematical Modelling and Numerical Simulation with Applications (MMNSA), 1(1), 11-23, (2021). [CrossRef]

[10] Naik, P.A., Owolabi, K.M., Yavuz, M. \& Zu, J. Chaotic dynamics of a fractional order HIV-1 model involving AIDS-related cancer cells. Chaos Solitons \& Fractals, 140, 110272, (2020). [CrossRef]

[11] Eskandari, Z., Alidousti, J., Avazzadeh, Z. \& Machado, J.T. Dynamics and bifurcations of a discrete-time prey-predator model with Allee effect on the prey population. Ecological Complexity, 48, 100962, (2021). [CrossRef]

[12] Naik, P.A., Yavuz, M. \& Zu, J. The role of prostitution on HIV transmission with memory: A modeling approach. Alexandria Engineering Journal, 59(4), 2513-2531,(2020). [CrossRef]

[13] Yavuz, M. European option pricing models described by fractional operators with classical and generalized Mittag-Leffler kernels. Numerical Methods for Partial Differential Equations, (2020). [CrossRef]

[14] Naik, P.A., Zu, J. \& Ghori, M.B. Modeling the effects of the contaminated environments on COVID-19 transmission in India.Results in Physics, 29, 104774, (2021). [CrossRef]

[15] Owolabi, K.M. \& Baleanu, D. Emergent patterns in diffusive Turing-like systems with fractional-order operator. Neural Computing and Applications, 1-18, (2021). [CrossRef]

[16] Joshi, H. \& Jha, B.K. Modeling the spatiotemporal intracellular calcium dynamics in nerve cell with strong memory effects. International Journal of Nonlinear Sciences and Numerical Simulation. [CrossRef]

[17] Owolabi, K.M., Karaagac, B. \& Baleanu, D. Dynamics of pattern formation process in fractional-order super-diffusive processes: a computational approach. Soft Computing, 1-18, (2021). [CrossRef]

[18] Dave, D.D. \& Jha, B.K. On finite element estimation of calcium advection diffusion in a multipolar neuron. Journal of Engineering Mathematics, 128(1), 1-15, (2021). [CrossRef]

[19] Alidousti, J., Eskandari, Z., Fardi, M. \& Asadipour, M. Codimension two bifurcations of discrete Bonhoeffer-van der Pol oscillator model. Soft Computing, 25(7), 5261-5276, (2021). [CrossRef] 
[20] Naik, P.A., Ghori, M.B., Zu, J., Eskandari, Z. \& Naik, M. Global dynamics and bifurcation analysis of a fractional-order SEIR epidemic model with saturation incidence rate. Mathematical Methods in the Applied Sciences, 45(3), 1-24, (2022). [CrossRef]

[21] Wang, J. \& Jia, Y. Analysis on bifurcation and stability of a generalized Gray-Scott chemical reaction model. Physica A: Statistical Mechanics and its Applications, 528, 121394, (2019). [CrossRef]

[22] Khan, A.Q. Neimark-Sacker bifurcation of a two-dimensional discrete-time chemical model. Mathematical Problems in Engineering, 2020, 3936242, (2020). [CrossRef]

[23] Naik, P.A., Eskandari, Z., Zu, J. \& Avazzadeh, Z. Multiple bifurcations of a discrete-time prey-predator model with mixed functional response. International Journal of Bifurcation and Chaos, 32(3), 1-15, (2022).

[24] Kuznetsov, Y.A. Elements of Applied Bifurcation Theory (Vol. 112). Springer Science \& Business Media, (2013).

[25] Eskandari, Z., Alidousti, J. \& Ghaziani, R.K. Codimension-one and -two bifurcations of a three-dimensional discrete game model. International Journal of Bifurcation and Chaos, 31(02), 2150023, (2021). [CrossRef]

[26] Kuznetsov, Y.A. \& Meijer, H.G. Numerical normal forms for codim 2 bifurcations of fixed points with at most two critical eigenvalues. SIAM Journal on Scientific Computing, 26(6), 1932-1954, (2005). [CrossRef]

[27] Govaerts, W., Ghaziani, R.K., Kuznetsov, Y.A. \& Meijer, H.G. Numerical methods for two-parameter local bifurcation analysis of maps. SIAM Journal on Scientific Computing, 29(6), 2644-2667, (2007). [CrossRef]

[28] Kuznetsov, I.A. \& Meijer, H.G.E. Numerical Bifurcation Analysis of Maps: From Theory to Software. Cambridge University Press, (2019).

Mathematical Modelling and Numerical Simulation with Applications (MMNSA) (http://www.mmnsa.org)

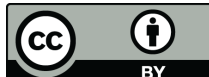

Copyright: () 2021 by the authors. This work is licensed under a Creative Commons Attribution 4.0 (CC BY) International License The authors retain ownership of the copyright for their article, but they allow anyone to download, reuse, reprint, modify, distribute, and/or copy articles in MMNSA, so long as the original authors and source are credited. To see the complete license contents, please visit (http://creativecommons.org/licenses/by/4.0/). 